\title{
BMJ Global Health Multiplexed technologies for sexually transmitted infections: global evidence on patient-centered and clinical health outcomes
}

\author{
Faheel Naeem, ${ }^{1,2}$ Angela Karellis, ${ }^{1,2}$ Suma Nair, ${ }^{3}$ Jean-Pierre Routy, ${ }^{4}$ \\ Cédric Philippe Yansouni, ${ }^{5,6}$ John Kim, ${ }^{7}$ Nitika Pai (1) ${ }^{1,2}$
}

\begin{abstract}
To cite: Naeem F, Karellis A, Nair S, et al. Multiplexed technologies for sexually transmitted infections: global evidence on patientcentered and clinical health outcomes. BMJ Global Health 2021;6:e005670. doi:10.1136/ bmjgh-2021-005670
\end{abstract}

Handling editor Seye Abimbola

FN and AK contributed equally.

Received 10 March 2021 Accepted 10 July 2021

Check for updates

C Author(s) (or their employer(s)) 2021. Re-use permitted under CC BY-NC. No commercial re-use. See rights and permissions. Published by BMJ.

For numbered affiliations see end of article.

Correspondence to Dr Nitika Pai;

Nitika.Pai@mcgill.ca

\section{ABSTRACT}

Introduction Conventional care packages around screening for sexually transmitted infections (STIs) entail multiple clinic visits and precipitate losses to follow-up. To prevent these losses, multiplexed technologies for STIs (immunochromatographic tests/devices/assays and molecular assays that can screen multiple pathogens or multiple strains of one STI) can yield same-day results in a single visit. Research evidence of patient-centred (preference, satisfaction) and clinical health outcomes (feasibility, case positivity, uptake, impact) has not been synthesised. We conducted a systematic review to fill this gap.

Methods For the period 2009-2020, two independent reviewers searched PubMed and Embase, retrieved 4440 citations and abstracted data from 42 relevant studies. Results Of 42 studies, 10 (23.8\%) evaluated multiplexed immunochromatographic and 32 (76.2\%) molecular assays. Outcomes were reported as follows: preference $(n=3)$, satisfaction $(n=2)$, uptake $(n=1)$, feasibility $(n=2)$, case positivity $(n=42)$ and impact $(n=11)$. Screened populations included various at-risk groups. A majority (86.1\%-92.4\%) of participants preferred $(60.2 \%-97.2 \%)$ multiplexed technologies (over conventional testing). Compared with conventional lab-based testing, test uptake improved by $99.4 \%$ (hepatitis C), $99.6 \%$ (Trichomonas vaginalis), $78.6 \%$ (hepatitis B) and $42.0 \%$ (HIV). Varying case positivities were documented depending on populations screened: HIV $(1.8 \%-29.3 \%)$, hepatitis B (1.1\%-23.9\%), hepatitis C $(0.5 \%-42.2 \%)$, Chlamydia trachomatis $(2.8 \%-30.2 \%)$, Neisseria gonorrhoeae $(0.0 \%$ $30.3 \%)$ and $T$. vaginalis $(0.0 \%-32.7 \%)$. Regarding impact, $70.0 \%-100.0 \%$ of screened participants were linked to care, with result turnaround times ranging from $14 \mathrm{~min}$ (immunochromatographic assays) to $300 \mathrm{~min}$ (molecular assays).

Conclusions Compared with conventional lab-based testing, rapid multiplexed technologies were preferred by testees and led to quicker turnaround times for many STIs yielding same-day results thereby allowing to initiate rapid linkages to care. They were further shown to be highly feasible and impactful for detection and treatment facilitation. Based on these promising results, multiplexed technologies offer potential to screen at-risk populations to reduce onward STI transmission worldwide.

\section{Key questions}

What is already known?

- A majority of sexually transmitted infections (STIS) are asymptomatic and if left undetected and untreated, they can lead to long-term health complications.

- Multiplexed technologies include both immunochromatographic tests and molecular assays.

What are the new findings?

- Multiplexed technologies were preferred by participants, operationally feasible, impacted detection and treatment of various STIs with same-day results and rapid linkages to care.

What do the new findings imply?

- Immunochromatographic and molecular assays are able to address gaps in the care cascade for screening and treating STIs.

- Our consolidation of research evidence on outcomes that are patient-centred and that can support implementation will aid a variety of stakeholders including healthcare professionals and policymakers.

\section{INTRODUCTION}

Diagnosing, treating and managing sexually transmitted infections (STIs) represent key pillars to reduce STI transmission and significant morbidity, and thus represent crucial targets across the spectrum of STI management. Approximately 1 million curable STIs are acquired globally each day leading to on average 376 million STIs being acquired annually worldwide, primarily in resource-limited settings. ${ }^{1}$ While a vast majority of these infections are asymptomatic, when left untreated, they can cause lifelong and often serious complications. Evidence from epidemiological studies has shown that commonly occurring STIs such as chlamydia, gonorrhoea and syphilis increase transmission of HIV. Moreover, behaviours associated with acquiring HIV infection increase the risk of acquiring 
additional STIs and of worsening severity among those with existing infections. ${ }^{2}$

STIs are conventionally diagnosed and confirmed using laboratory-based tests, considered the reference (gold standard) on account of their high diagnostic accuracy. ${ }^{34}$ However, they include culture, often entail multiple patient visits due to the longer turnaround time to test results (at minimum two visits: to collect samples and to communicate test results to individuals), and sample transportation; collectively, these multiple requirements associated with lab-based testing precipitate inaction and consequent losses to follow-up. ${ }^{45}$ Furthermore, they often require substantial laboratory infrastructure used by trained laboratory personnel making them difficult to carry out in remote settings. This puts rural communities with high rates of STI transmission at risk. ${ }^{6-8}$ With a rise in the global prevalence of STIs, a shift towards efficient technologies such as multiplexed technologies is needed to enable healthcare providers to screen several STIs both rapidly and accurately and return the result to the patient often in one visit.

Multiplexed rapid screening technologies are of two types primarily: (a) antibody-based immunochromatographic tests/assays/handheld devices and (b) molecular tests/assays. Multiplexed technologies also meet the needs and preferences of testees, primarily by reducing the number of clinic visits, with incumbent time and cost savings. As a result, multiplexed rapid testing, if integrated into routine testing, can optimise treatment linkages thereby minimising losses to follow-up and patient anxiety associated with conventional STI testing. ${ }^{9}$

While a number of published STI-related reviews have described technologies and their use both in clinical and field settings worldwide, ${ }^{10-12}$ a systematic review of evidence to support implementation (ie, impact, uptake, feasibility) and to collect end users' preferences beyond diagnostic accuracy has not been synthesised. With a view to plug the knowledge gap, we conducted a systematic review. Diagnostic accuracy outcomes (sensitivity, specificity) have been separately synthesised and are being peer reviewed. ${ }^{13}$

\section{METHODS}

\section{Search strategy and study selection}

We followed Preferred Reporting Items for Systematic Reviews and Meta-Analyses (http://www.prisma-statement.org/). We registered the protocol with The International Prospective Register of Systematic Reviews (registration number: CRD4202179218). ${ }^{14}$

For the period 1 January 2009-20 April 2020, two independent reviewers (FN and $\mathrm{AK}$ ) searched two electronic databases, PubMed and Embase, to retrieve relevant primary articles and conference abstracts. In addition, we searched bibliographies of included studies. We included abstracts only if full-text articles were unavailable.

Our objective was to consolidate evidence on clinical outcomes pertaining to multiplexed technologies,

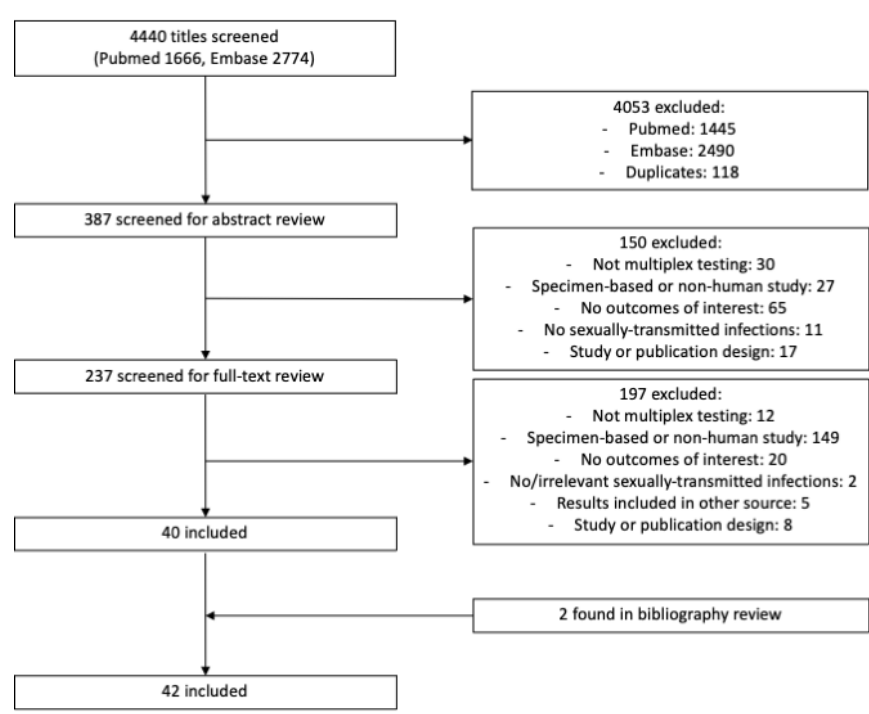

Figure 1 Preferred Reporting Items for Systematic Reviews and Meta-Analyses flow chart.

including immunochromatographic point-of-care devices and molecular assays used to screen populations for various bacterial, viral and parasitic STIs. FN and AK independently reviewed all citations to achieve a final subset of studies for inclusion (figure 1). Patients or the public were not involved in the design, or conduct, or reporting, or dissemination plans of our research.

\section{Search string}

Our search string included multiplex*, duplex*, triplex*, quadruplex*, simultaneous*, point-of-care, rapid, platform, molecular, sexually transmitted infection*, sexually transmitted disease*, human immunodeficiency virus, acquired immunodeficiency syndrome, chlamydia, gonorrh*, hepatitis, syphilis, Treponema pallidum, human papillomavirus, herpes simplex and trichomonas.

\section{Eligibility criteria}

Studies that were included for review consisted of fulltext primary articles or abstracts that assessed rapid multiplexed technologies where multiplexed was defined as testing for more than one STI or more than one strain of the same STI. Reviews, reports, editorials, commentaries and studies that used precollected specimens (not for the purposes of STI testing) or studies not based on human subjects were excluded (figure 1).

\section{Outcomes}

Four outcomes were analysed: impact, feasibility and preference, and the number of positive cases for each STI.

The impact of multiplexed technologies included their ability to detect new infections, turnaround times to test results and linkages to care. A change in uptake was also used to assess impact; this was defined as the increase in STI testing once multiplexed rapid testing was made available and quantified by calculating the absolute difference between the percentage of test usage from baseline, 
that is, conventional lab-based testing, to follow-up, that is, multiplexed rapid testing.

Testee preference was assessed in one of several ways, depending on the manner reported in each article: (1) participants' preference to undergo multiplexed testing using a rapid test (immunochromatographic or molecular assay) over conventional testing; (2) participants' satisfaction or acceptance of multiplexed technologies; and (3) participants' willingness to recommend multiplexed testing to others. Preference outcomes were quantified by dividing the number of participants who indicated preference of rapid multiplexed testing by the total number of participants who were surveyed. Feasibility was quantified by metrics such as completion rate of the multiplex rapid testing strategy.

Finally, we aimed to identify the case positivity of the specific STIs based on the positive test results generated by multiplexed technologies. The number of positive STI cases was ascertained by determining the number of laboratory-confirmed positive cases divided by the total number of participants tested for the specific STI. When available, the positive (PPV) or negative (NPV) predictive value of each index test used to ascertain STI positivity was recorded or calculated.

\section{Data abstraction}

The data abstraction was performed independently by two reviewers (FN and AK). Abstraction items were tabulated in a data abstraction form and included general study characteristics, participant information, types of index and reference tests, diagnostic accuracy and the above-stated four outcomes.

\section{Quality assurance}

The quality of included studies was assessed using a revised tool for the Quality Assessment of Diagnostic Accuracy Studies (QUADAS-2) by the two reviewers (FN and $\mathrm{AK}) .^{15}$

\section{RESULTS}

As shown in figure 1, we reviewed a total of 4440 citation titles. After deduplication and our initial review of titles, we reviewed 387 abstracts. Of 387, 150 citations were excluded such that a total of 237 citations were eligible for full-text review. Of 237 citations, 197 did not meet our eligibility criteria, leading to 40 eligible publications. Two additional sources were identified from bibliography review and added to the final set, therefore a total of 42 publications were included in the final set. The reasons of exclusion as well as the counts for each are detailed in figure 1. Online supplemental table 1 provides a description (including author/year of publication; study design, setting and population; STIs and the type of diagnostic test used) of the 42 studies included.

\section{Description of included studies}

Of the 42 studies included, 10 (23.8\%) studies reported the use of multiplexed immunochromatographic tests, while $32(76.2 \%)$ studies reported using multiplexed molecular assays (online supplemental table 1 ).

Our review includes data from high-income, middleincome and low-income countries and includes key populations at a higher risk of acquiring STIs (such as men who have sex with men (MSM), sex workers, injection drug users (IDUs) as well general STI clinic attendees).

All $42(100.0 \%)$ studies reported on case positivity. ${ }^{351617}$ About 11 studies reported on impact outcomes; 3 (7.1\%) studies reported on preference ${ }^{17-19} ; 2(4.8 \%)$ on patient satisfaction $^{19}{ }^{20} ; 2(4.8 \%)$ on acceptance of multiplexed testing ${ }^{1718}$ and $1(2.9 \%)$ on recommending multiplexed testing $^{19}$ (table 1 ).

\section{Impact}

The impact of multiplexed technologies was defined in one of several ways including their ability to detect new infections, increase uptake and to improve turnaround times to test results and linkages to care. Impact was reported by $11(25.6 \%)$ where $3(6.8 \%)$ studies reported on the increased detection of new/previously undiagnosed infections as a result of multiplexed testing, 1 $(2.3 \%)$ study reported the increase in multiplexed testing from baseline ${ }^{21} 6(13.9 \%)$ studies reported the turnaround time to test results, ${ }^{22-27}$ and $3(6.9 \%)$ reported on linkages to/retention in care ${ }^{182128}$ (table 1).

One study reported the detection of a single new infection of syphilis and HIV using an immunochromatographic test, respectively. ${ }^{19}$ In another study, 30 new infections of hepatitis B virus (HBV) and 11 new infections of Trichomonas vaginalis (TV) were detected with immunochromatographic assays. ${ }^{21}$ Finally, one study determined that $3.2 \%$ more infections of TV were detected by a molecular assay than culture and $71.4 \%$ more infections were detected compared with wet mount ${ }^{29}$ (table 1 ).

Increased uptake of multiplexed testing from baseline was reported for four STIs: HIV $(58.0 \%$ at baseline to $100.0 \%$ at follow-up, an overall $42.0 \%$ increase), HBV $(21.0 \%$ at baseline and $100.0 \%$ at follow-up, an overall $78.6 \%$ increase), hepatitis $\mathrm{C}$ virus (HCV) $(0.6 \%$ at baseline and $100.0 \%$ at follow-up, an overall $99.4 \%$ increase) and TV $(0.4 \%$ at baseline and $100.0 \%$ at follow-up, an overall $99.6 \%$ increase). ${ }^{21}$ The turnaround time for immunochromatographic devices ranged from $15 \mathrm{~min}$ to $20 \mathrm{~min}$, whereas for molecular assays it ranged from $14 \mathrm{~min}$ to $300 \mathrm{~min}^{22-27}$ Most participants who underwent multiplexed testing were linked to care $(70.0 \%-$ $100.0 \%)^{182128}$ (table 1).

\section{Preference and feasibility}

Testee preference for multiplexed testing varied from a low of $60.2 \%$ to a high of $97.2 \%$ among study participants. ${ }^{18} 1921$ Overall, participants reported high satisfaction with being tested by multiplexed technologies $(92.0 \%-99.5 \%)^{19}$ and high acceptance of multiplexed technologies $(100.0 \%){ }^{18}{ }^{21}$ With regard to 
Table 1 Impact outcomes (detection of new infections/uptake/turnaround time/linkage to care) associated with the use of rapid multiplexed STI diagnostic devices

\begin{tabular}{|c|c|c|c|}
\hline Author, year & Impact outcome & Multiplex test type & Result \\
\hline Pai et al, $2014^{19}$ & New infection & IMT & $\begin{array}{l}1 / 109(0.9 \%) \text { new infection of syphilis and } \\
1 / 109(0.9 \%) \text { new infection of HIV detected with } \\
\text { IMT }\end{array}$ \\
\hline Pai et al, 2014*19 & New infection & IMT & $\begin{array}{l}56 / 375 \text { (14.9\%) diagnosed with HIV, } 75 / 375 \\
\text { (20.0\%) with HBV, }(37 / 375) 9.9 \% \text { with syphilis, } \\
2 / 375 \text { (0.5\%) with HCV }\end{array}$ \\
\hline Van Der Pol et al, $2017 \dagger^{29}$ & New infection & Molecular assay & $\begin{array}{l}3.2 \% \text { more infections of TV detected by } \\
\text { molecular assay than culture and } 71.4 \% \text { more } \\
\text { than wet mount }\end{array}$ \\
\hline Pant Pai et al, $2019^{21}$ & Uptake & $\mathrm{IMT}$ & $\begin{array}{l}\text { 99.4\% increase for HCV IMT } \\
79.0 \% \text { increase for HBV IMT } \\
42.0 \% \text { increase for HIV IMT } \\
99.6 \% \text { increase for TV IMT }\end{array}$ \\
\hline Le Roy et al, $2012^{23}$ & TAT & Molecular assay & 4.5-5 hours for 50 samples (CT) \\
\hline Longo et al, $2018^{24}$ & TAT & IMT & 15 min (HIV, HBV and HCV) \\
\hline Mboumba Bouassa et al, 2018 & TAT & $\mathrm{IMT}$ & $15 \min (\mathrm{HIV}, \mathrm{HBV}, \mathrm{HCV})$ \\
\hline Nuñez-Forero et al, $2016^{26}$ & TAT & Molecular assay & $14 \min (\mathrm{CT}$ and $\mathrm{NG})$ \\
\hline Omoding et al, $2014^{27}$ & TAT & $\mathrm{IMT}$ & 20 min (syphilis and HIV) \\
\hline Causer et al, $2015^{22}$ & TAT & Molecular assay & $91 \min (\mathrm{CT}$ and $\mathrm{NG})$ \\
\hline Pant Pai et al, $2019^{21}$ & Retention in care & $\mathrm{IMT}$ & $\begin{array}{l}95.0 \% \text { patients retained in care (HIV, HBV, HCV, } \\
\text { TV) }\end{array}$ \\
\hline Pant Pai et al, $2019^{21}$ & Linkage to care & $\mathrm{IMT}$ & $\begin{array}{l}70.0 \% \text { patients linked to care (HIV, HBV, HCV, } \\
\text { TV) }\end{array}$ \\
\hline
\end{tabular}

*Study conducted in Canadian and Indian populations.

†Raw data were unavailable in the publication.

CT, Chlamydia trachomatis; HBV, hepatitis B virus; HCV, hepatitis C virus; IMT, immunochromatographic test; NG, Neisseria gonorrhoeae;

STI, sexually transmitted infection; TAT, turnaround time; TV, Trichomonas vaginalis.

recommendation, up to $99.1 \%$ of study participants would recommend multiplexed technologies to others. ${ }^{19}$

Finally, with respect to feasibility, two $(4.7 \%)$ studies reported on completion rates ${ }^{1921}$ (table 2). In terms of feasibility, among participants, completion rate of multiplexed testing procedures ranged between $86.1 \%$ and $92.4 \%^{19}{ }^{21}$ (table 2).

\section{Positivity (case), PPV and NPV}

Varying prevalence rates of STIs were documented in studies, conducted in diverse populations; we computed PPVs and NPVs across studies and technologies (both molecular and immunochromatographic assays) in online supplemental tables 2 and 3.

The high PPVs and NPVs of both immunochromatographic tests and molecular assays support the usage of multiplexed technologies in the detection of numerous STIs whether disease prevalence is low or high in a variety of populations and settings worldwide.
For molecular assays, the ranges of PPVs and NPVs for assessing STIs were calculated or abstracted. These included: Chlamydia trachomatis $(91.2 \%-100.0 \%$ and 98.5\%-100.0\%, respectively), Neisseria gonorrhoeae (NG) (50.0\%-100.0\% and $98.4 \%-100.0 \%$, respectively), TV $(37.5 \%-100.0 \%$ and $98.9 \%-100.0 \%$, respectively), Treponema pallidum (syphilis) (100.0\% and 93.3\%-100.0\%, respectively), herpes simplex virus (HSV)-1 (75.0\%$100.0 \%$ and $12.5 \%-100.0 \%$, respectively), and HSV-2 (40.0\%-100.0\% and $36.1 \%-100.0 \%$, respectively).

Similarly, for immunochromatographic tests, the ranges of PPVs and NPVs for assessing the most commonly reported STIs were: T. pallidum $(93.3 \%-100.0 \%$ and $86.0 \%-100.0 \%$, respectively), HCV $(97.1 \%-100.0 \%$ and $95.7 \%-98.2 \%$, respectively), and HIV $(94.1 \%-100.0 \%$ and $99.5 \%-100.0 \%$, respectively).

Twenty pathogens were identified by these tests. These included: adenovirus, C. trachomatis, Gardnerella vaginalis, 


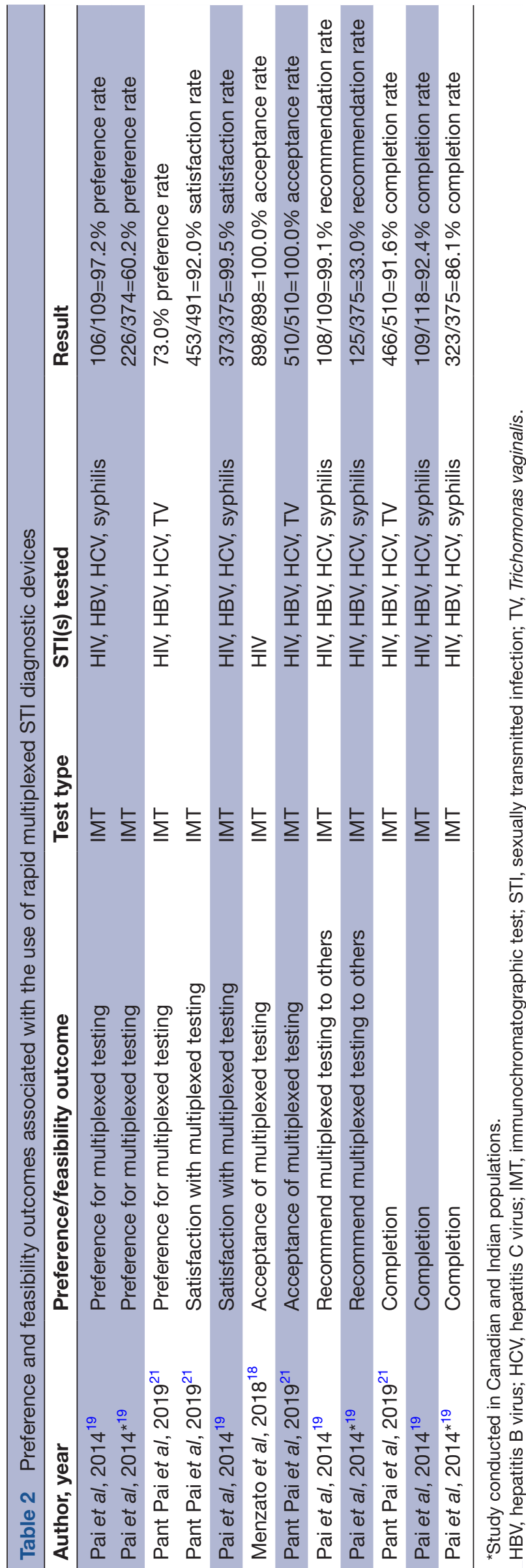

Haemophilus ducreyi, H. influenzae, HBV, HCV, HIV, HSV-1 and HSV-2, human papillomavirus (HPV), Mycoplasma genitalium, M. hominis, NG, N. meningitidis, Streptococcus pneumoniae, T. pallidum (syphilis), TV, Ureaplasma parvum, U. urealyticum and other Ureaplasma spp.

Across studies, the most commonly reported STIs were: C. trachomatis in $22(51.1 \%)$ studies, NG in $21(48.8 \%)$, TV in $11(25.6 \%)$, HIV in $6(13.9 \%)$, T. pallidum in 6 (13.9\%), HSV-1/2 in $8(18.6 \%), \mathrm{HBV}$ in $3(6.9 \%)$, and HCV in $4(9.3 \%)$ studies, respectively.

For the more commonly reported STIs, case positivity estimates varied within populations studied: $C$. trachomatis (2.8\%-30.2\%), NG (0.3\%-30.3\%), TV $(0.1 \%-32.7 \%)$, T. pallidum (syphilis) $(0.9 \%-27.0 \%), \mathrm{HSV}-1 / 2 \quad(0.8 \%-$ $90.2 \%)$, HCV $(0.5 \%-42.2 \%)$, HIV (1.8\%-29.9\%), HBV $(1.1 \%-23.9 \%)$, and HPV $(12.3 \%-98.2 \%)$.

\section{Quality assessment of included studies}

The QUADAS-2 checklist was used to evaluate quality of the included studies. In general, the majority of studies $(83.7 \%)$ used appropriate reference tests to ascertain patient disease status, and most studies $(60.5 \%)$ included all patients in their respective analyses. Figure 2 provides a breakdown of the included studies according to the quality checklist.

\section{DISCUSSION}

Multiplexed technologies fill many gaps in the spectrum of STI diagnostic care. ${ }^{240}$ These technologies offer the ability to screen for many pathogens including those that do not present any clinical manifestations and those that are not commonly identified in standard STI diagnostic practices yet are able to give rise to coinfections. The test results also help catalyse the process of diagnosis and the process of seeking subsequent linkages to care. In fact, modelling studies have shown that immunochromatographic tests with adequate sensitivity can reduce prevalence of disease in communities where it is otherwise high. ${ }^{22}$ Particularly now, during the COVID-19 pandemic, rapid testing is more relevant than ever and would offer several benefits, such as to destigmatise testing, whether related to STI or COVID-19 testing, and to expedite turnaround time for both types of pathogens. Certain multiplexed test devices are now available that can test for both COVID-19 and STIs, such as Cepheid's GeneXpert. $^{31} 32$ The ability to conduct simultaneous testing of various pathogens presents as an additional advantage in diagnostic evaluations. ${ }^{33}$

The evidence presented herein suggests that over the past decade, multiplexed technologies are increasingly being developed and used to address the burden of the most common viral, bacterial and parasitic STIs in both high-income and low-income countries. While we found high feasibility of execution of multiplexed rapid testing (86.1\%-92.4\%), ${ }^{419}{ }^{21}$ the uptake of these technologies for less commonly screened STI was very high: HBV (79.0\%), HCV (99.4\%) and TV (99.6\%). The 


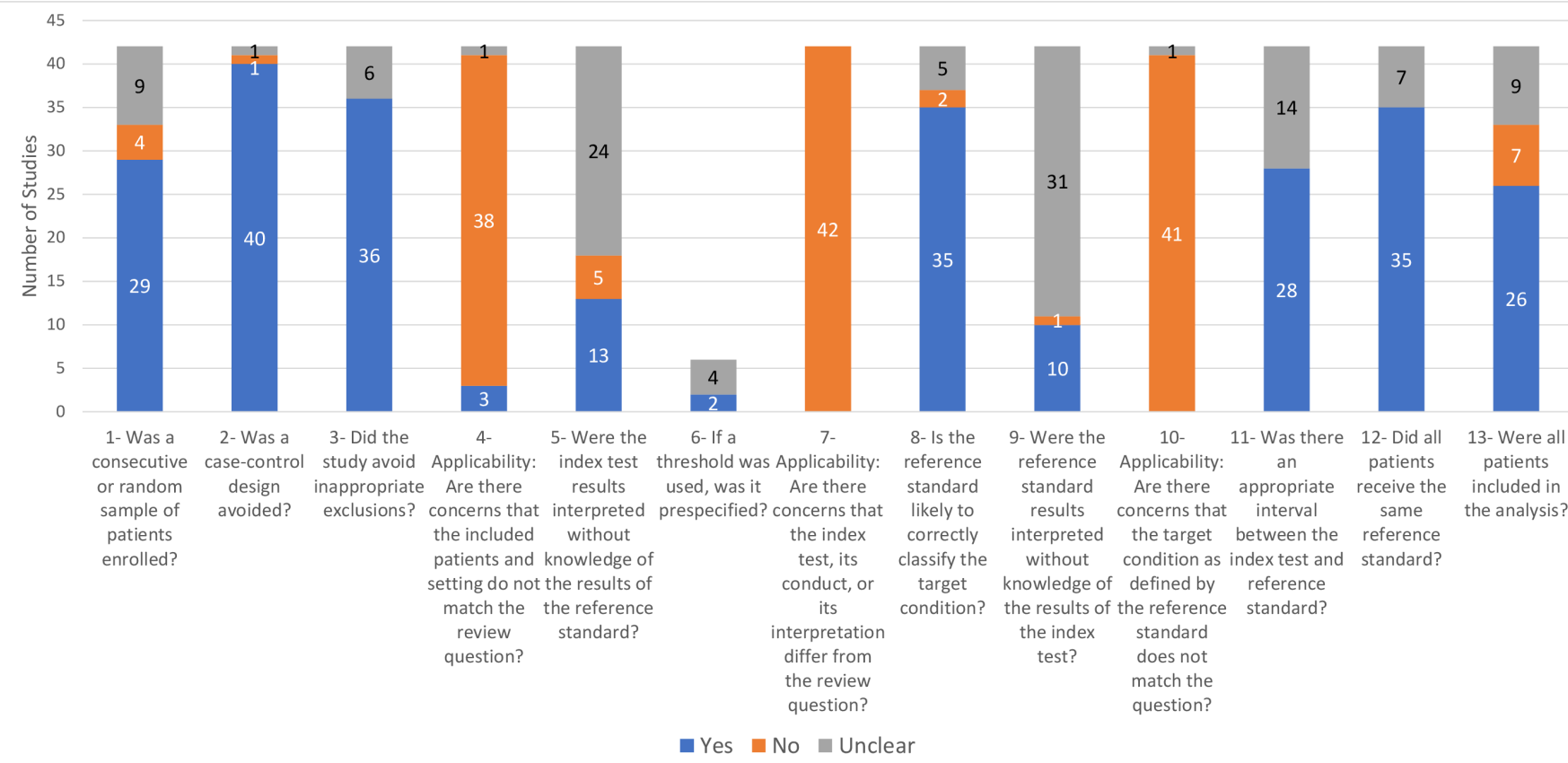

Figure 2 Quality assessment of included studies.

high PPVs and NPVs of both immunochromatographic and molecular assays support the usage of multiplexed technologies in the detection of numerous STIs whether disease prevalence is low or high in a variety of populations and settings worldwide. Unsurprisingly, with respect to case positivity and new cases detected with these multiplexed technologies, we found that the highest rates were reported for asymptomatic C. trachomatis, NG, T. pallidum and TV. ${ }^{16}{ }^{34-36}$ Our findings suggest that asymptomatic STIs may be left undetected and untreated thereby emphasising the need for routine STI screening for at-risk populations, including MSM, IDUs, sex workers and transgender populations residing in endemic settings. Given their high feasibility and rapid turnaround time, multiplexed technologies have the potential to accelerate the screening and treatment process of these key populations and other asymptomatic individuals. ${ }^{37}$ These results further support implementation of multiplexed rapid tests in clinical care, particularly as 41 out of the 42 studies included in our final set were observational in nature and therefore alluded to real-world implementation of these test devices.

We also found evidence in favour of rapid turnaround time, and that multiplexed rapid testing was preferred by participants over conventional lab-based testing $(60.2 \%-$ 97.2\%). Published findings corroborate these results; Rompalo et al and Widdice et al reported that patients are in favour of rapid and user-friendly diagnostic tests. ${ }^{12} 38$ With respect to HIV, multiplexed technologies addressed various barriers to testing including having to wait for test results. ${ }^{39}$ Long wait times can induce feelings of prolonged anxiety and fear among test seekers thereby discouraging individuals from seeking testing.
As the turnaround times ranged between 15 and $20 \mathrm{~min}$ for immunochromatographic devices and between $14 \mathrm{~min}$ and 5 hours for molecular assay-based testing, rapid tests have the potential to eliminate the time barrier in getting tested for STIs by yielding same-day results. While a 5-hour turnaround time for a molecular assay may be considered a lengthy period to receive test results, it nonetheless obviates the need for additional patient visits, therefore facilitating the testing and linkage to care process for individuals with limited healthcare access, such as those residing in rural areas.

Additional published findings further support the feasibility and usefulness of rapid testing into clinical care. For instance, in a qualitative research study, Fuller $e t$ al concluded that patients and clinicians across six sexual health clinics in the UK expressed their acceptance in regard to point-of-care testing, particularly if information is provided prior to testing regarding the changes they may expect in services rendered.$^{40}$ Furthermore, Harding-Esch et al demonstrated the high feasibility associated with a 'sample first' clinical pathway where patients provided samples on arrival at a London sexual health clinic, subsequently to be tested in the point of care. Their findings illustrated that over $90 \%$ of patients reported high satisfaction with the evaluated strategy, and that all results were available prior to patients leaving the clinic which led to high linkage to treatment rates. ${ }^{41}$

Despite the benefits associated with rapid multiplex testing, we must however acknowledge that certain devices are not fully optimisable at the point of care. While the design of handheld immunochromatographic tests fully support point-of-care usage, the majority of molecular tests are conducive for laboratory testing. The 
sole molecular assay recommended for use near testees is the Cepheid GeneXpert. ${ }^{22} 42-45$

\section{Strengths and limitations}

We were unable to conduct a meta-analysis due to the heterogeneity of settings, populations and pathogens screened and outcomes that could not be pooled due to the lack of data in clinically relevant subgroups defined by pathogens, populations and technologies. This limitation needs to be addressed in future research. Moreover, the wide range of pathogens evaluated across studies present as a limitation and as a strength. A number of studies assessed STIs that are not generally included in STI testing guidelines such as various Mycoplasma and Ureaplasma spp. As a result, these infections were omitted from analysis as they demonstrate limited clinical utility. Also, while our review focused on the preference, case positivity, uptake, feasibility and impact of multiplexed rapid testing, the examination of these devices to detect antimicrobial resistance may be warranted, in particular with respect to gonorrhoea testing.

Study limitations, as identified by the study authors, included variability in disease prevalence in the settings and populations screened that led to limited case finding. ${ }^{19} 26$ 46-48 Convenience sampling potentially introduced biases (namely, volunteer, selection and/or confounding), ${ }^{192149}$ and missing data generated potential for information bias. ${ }^{50}$ Technological challenges were reported in regard to the multiplexed devices. For instance, the simultaneous molecular amplification performed by STDFinder used by Muvunyi et al reduced the device's capability to amplify and detect singleton targets. ${ }^{51}$ Molecular assays reported PCR drift. ${ }^{52}$ Moreover, skilled healthcare staff were required to perform testing with molecular assays and venous blood was required for confirmatory tests. ${ }^{19} 272946$

\section{Implications}

Several important implications of timely screening ensue as a result of ascertaining disease status early: the mitigation of transmission of disease and disease-specific complications, such as chronic pelvic pain, ectopic pregnancies, stillbirths, infertility, hepatic failure or cirrhosis. ${ }^{19} 53$ These time savings allow people who test positive to immediately be linked to counselling and care, as evidenced by the $70.0 \%-100.0 \%$ of tested patients in three identified studies being linked to care after multiplexed rapid testing. ${ }^{18128}$ An additional benefit of using these tests is the non-invasive nature of specimen collection, such as blood samples. While our findings support the numerous clinical benefits associated with rapid multiplexed devices in STI management, the importance of conventional lab-based testing should not be overlooked to confirm preliminary positive test results as these still constitute the gold standard of testing to diagnose STIs.

Our systematic review findings add value to the current body of literature as it consolidates data relevant for healthcare professionals, policymakers, decision makers, and government officials, academics and researchers to make decisions on an offer of multiplexed testing to meet the needs of integrated testing agenda of public health organisations.

\section{CONCLUSION}

With the issues related to routine STI testing, particularly the high rates of loss to follow-up and the lengthy turnaround time to test results, multiplexed rapid testing (both immunochromatographic and molecular assays) offer the potential to fill many early and timely screening gaps in the spectrum of care.

We conclude that both multiplexed technologies were found to be feasible and preferred by participants, impacted detection and treatment of many STIs, with provision of same-day test results and rapid linkages to care. Given the increasing incidence of STIs worldwide, multiplexed technologies can safely be the future of integrated screening initiatives for STI diagnosis and treatment worldwide. Based on these findings, we recommend the incorporation of multiplex rapid tests into clinical care, whether they may be in the form of immunochromatographic or molecular assays. Testees' high satisfaction of multiplexed rapid testing as well as the added benefit and impact support their usage in the spectrum of STI diagnostic care, particularly to complement conventional lab-based testing which may at times present as a suboptimal testing approach to reach vulnerable at-risk populations.

\section{Author affiliations}

${ }^{1}$ Department of Medicine, McGill University, Montreal, Quebec, Canada ${ }^{2} \mathrm{CORE}$, Research Institute of the McGill University Health Centre, Montreal, Quebec, Canada

${ }^{3}$ Community Medicine, Kasturba Medical College Manipal, Manipal, Karnataka, India

${ }^{4}$ Chronic Viral IIIness Service, McGill University Health Centre, Montreal, Quebec, Canada

${ }^{5}$ J D MacLean Centre for Tropical Diseases, McGill University Health Centre, Montreal, Quebec, Canada

${ }^{6}$ Division of Infectious Diseases and Department of Microbiology, McGill University Health Centre, Montreal, Quebec, Canada

${ }^{7}$ National Laboratory for HIV Reference Services, Public Health Agency of Canada, Winnipeg, Quebec, Canada

Twitter Nitika Pai @nikkiannike

Acknowledgements The reviewers ( $F N$ and $A K$ ) acknowledge the support of Ms Genevieve Gore, librarian at McGill University, for her help in conducting the literature search.

Contributors FN, AK and NP designed, drafted and reviewed the initial manuscript, while the remaining authors (SN, J-PR, CPY, JK) provided critique on subsequent drafts. The search strategy was developed and executed by FN, AK and NP. The quality assessment was performed by FN and AK. All authors approved and contributed to the final written manuscript.

Funding The authors acknowledge the support of the following agencies: the Canadian Institutes of Health Research grant PJT 153149 (NP), the Fonds de recherche du Québec-Santé Research-Scholar Senior Award (NP), the MUHC Foundation (NP) and the India-Canada Centre for Innovative Multidisciplinary Partnerships to Accelerate Community Transformation and Sustainability (IC-IMPACTS) (NP and SN). 
Disclaimer The agencies had no role in study design, data collection and analysis, decision to publish, or preparation of the manuscript.

Competing interests None declared.

Patient consent for publication Not required.

Provenance and peer review Not commissioned; externally peer reviewed.

Data availability statement All data relevant to the study are included in the article or uploaded as supplemental information.

Supplemental material This content has been supplied by the author(s). It has not been vetted by BMJ Publishing Group Limited (BMJ) and may not have been peer-reviewed. Any opinions or recommendations discussed are solely those of the author(s) and are not endorsed by BMJ. BMJ disclaims all liability and responsibility arising from any reliance placed on the content. Where the content includes any translated material, BMJ does not warrant the accuracy and reliability of the translations (including but not limited to local regulations, clinical guidelines, terminology, drug names and drug dosages), and is not responsible for any error and/or omissions arising from translation and adaptation or otherwise.

Open access This is an open access article distributed in accordance with the Creative Commons Attribution Non Commercial (CC BY-NC 4.0) license, which permits others to distribute, remix, adapt, build upon this work non-commercially, and license their derivative works on different terms, provided the original work is properly cited, appropriate credit is given, any changes made indicated, and the use is non-commercial. See: http://creativecommons.org/licenses/by-nc/4.0/.

ORCID iD

Nitika Pai http://orcid.org/0000-0002-4672-0500

\section{REFERENCES}

1 World Health Organization. Report on global sexually transmitted infection surveillance, 2018

2 Gore-Felton C, Vosvick M, Bendel T, et al. Correlates of sexually transmitted disease infection among adults living with HIV. Int J STD AIDS 2003;14:539-46.

3 Fernández G, Martró E, González V, et al. Usefulness of a novel multiplex real-time PCR assay for the diagnosis of sexuallytransmitted infections. Enferm Infecc Microbiol Clin 2016;34:471-6.

4 Pant Pai N, Behlim T, Landry G. Will a quad point-of-care multi plexed assay for HIV, HCV, HBV, syphilis be feasible, accurate and preferred by injection drug users: a pilot study from Montreal, Canada. Can J Infect Dis Med Microbiol 2013;SA:88A-9.

5 Gimenes F, Medina FS, Abreu ALPde, et al. Sensitive simultaneous detection of seven sexually transmitted agents in semen by multiplex-PCR and of HPV by single PCR. PLoS One 2014;9:e98862.

6 Kozel TR, Burnham-Marusich AR. Point-Of-Care testing for infectious diseases: past, present, and future. $J$ Clin Microbiol 2017;55:2313-20.

7 Oliff M, Mayaud P, Brugha R, et al. Integrating reproductive health services in a reforming health sector: the case of Tanzania. Reprod Health Matters 2003;11:37-48.

8 Petti CA, Polage CR, Quinn TC, et al. Laboratory medicine in Africa: a barrier to effective health care. Clin Infect Dis 2006;42:377-82.

9 Tucker JD, Bien CH, Peeling RW. Point-Of-Care testing for sexually transmitted infections: recent advances and implications for disease control. Curr Opin Infect Dis 2013;26:73-9.

10 Adamson PC, Loeffelholz MJ, Klausner JD. Point-Of-Care testing for sexually transmitted infections: a review of recent developments. Arch Pathol Lab Med 2020;144:1344-51.

11 Cristillo $A D$, Bristow CC, Peeling R, et al. Point-Of-Care sexually transmitted infection diagnostics: proceedings of the StAR sexually transmitted Infection-Clinical trial group programmatic meeting. Sex Transm Dis 2017;44:211-8.

12 Rompalo AM, Hsieh Y-H, Hogan T, et al. Point-of-care tests for sexually transmissible infections: what do 'end users' want? Sex Health 2013:10:541-5

13 Diagnostic performance of multiplexed. Platform and point-of-care rapid tests for HIV and sexually-transmitted blood-borne infections: a systematic review. 23rd International AIDS Conference [Virtual]; 6-10 July 2020, 2020.

14 Karellis A, Naeem F, Mallya SD. Evaluation of rapid multiplex diagnostic devices for sexually-transmitted infections: a systematic review. Prospero 2020 CRD42020179218. Available: https://www. crd.york.ac.uk/prospero/display record.php?ID=CRD42020179218 [Accessed 30 July 2020].
15 Whiting P, Rutjes AWS, Reitsma JB, et al. The development of QUADAS: a tool for the quality assessment of studies of diagnostic accuracy included in systematic reviews. BMC Med Res Methodol 2003;3:25.

16 Berçot B, Amarsy R, Goubard A, et al. Assessment of coinfection of sexually transmitted pathogen microbes by use of the Anyplex II STI-7 molecular kit. J Clin Microbiol 2015;53:991-3.

17 Zhao Y, Cao X, Tang J, et al. A novel multiplex real-time PCR assay for the detection and quantification of HPV16/18 and HSV1/2 in cervical cancer screening. Mol Cell Probes 2012;26:66-72.

18 Menzato F, Bosa L, Sifna A. Successful simultaneous screening of sickle cell disease, HIV and tuberculosis in rural guinea bissau, West Africa through rapid tests and a standardized clinical questionnaire: an outreach program due to a public-private partnership. Blood Conference: 60th Annual Meeting of the American Society of Hematology, ASH;132(Suppl. 1), 2018.

19 Pai NP, Dhurat R, Potter M, et al. Will a quadruple multiplexed pointof-care screening strategy for HIV-related co-infections be feasible and impact detection of new co-infections in at-risk populations? Results from cross-sectional studies. BMJ Open 2014:4:e005040.

20 Parnell B, Tong W, Menon-Johansson A. Has the introduction of a multiplex PCR for herpes simplex viruses and Treponema pallidum impacted the patient journey for those diagnosed with primary syphilis? HIV Medicine 2014;3:112.

21 Pant Pai N, Daher J, Prashanth HR, et al. Will an innovative connected AideSmart! app-based multiplex, point-of-care screening strategy for HIV and related coinfections affect timely quality antenatal screening of rural Indian women? Results from a crosssectional study in India. Sex Transm Infect 2019;95:133-9.

22 Causer LM, Hengel B, Natoli L, et al. A field evaluation of a new molecular-based point-of-care test for Chlamydia and gonorrhoea in remote Aboriginal health services in Australia. Sex Health 2015;12:27-33.

23 Le Roy C, Le Hen I, Clerc M, et al. The first performance report for the Bio-Rad DX CT/NG/MG assay for simultaneous detection of Chlamydia trachomatis, Neisseria gonorrhoeae and Mycoplasma genitalium in urogenital samples. J Microbiol Methods 2012;89:193-7.

24 Longo JDD, Mboumba Bouassa R-S, Mbeko Simaleko M, et al. Usefulness of simultaneous screening for HIV-specific and HCVspecific antibodies and HBsAg by a capillary-based multiplex rapid diagnostic test to strengthen linkage-to-care in sub-Saharan patients attending sexually transmitted infection clinic. J Med Virol 2018:90:1549-52.

25 Mboumba Bouassa R-S, Nodjikouambaye ZA, Sadjoli D, et al. Usefulness of simultaneous screening for HIV- and hepatitis C-specific antibodies and hepatitis B surface antigen by capillarybased multiplex immunochromatographic rapid test to strengthen prevention strategies and linkage to care in Childbearing-Aged women living in resource-limited settings. Open Forum Infect Dis 2018;5:ofy069.

26 Nuñez-Forero L, Moyano-Ariza L, Gaitán-Duarte H, et al. Diagnostic accuracy of rapid tests for sexually transmitted infections in symptomatic women. Sex Transm Infect 2016;92:24-8.

27 Omoding D, Katawera V, Siedner M, et al. Evaluation of the SD Bioline HIV/Syphilis Duo assay at a rural health center in southwestern Uganda. BMC Res Notes 2014:7:746.

28 Kalla GCM, Voundi EV, Guiadem R, et al. Mass campaigns for HIV, HBV (HBsAg) and HCV screening by multiplex rapid diagnostic test in sub-Saharan Africa using mobile units: the game changer. Int $J$ Infect Dis 2019;79:107.

29 Van Der Pol B, Williams JA, Fuller D, et al. Combined testing for Chlamydia, gonorrhea, and Trichomonas by use of the BD max CT/ GC/TV assay with genitourinary specimen types. $J$ Clin Microbiol 2017:55:155-64.

30 Mawu F, Davies SC, McKechnie M. Sexually transmitted infections among female sex workers in Manado, Indonesia using a multiplex PCR. Sex Health 2009;6:371-2.

31 Cepheid. Xpert@ Xpress SARS-CoV-2. Available: https://www. cepheid.com/en/about/SARS-CoV-2-Test-Development-Information [Accessed 3 May 2021]

32 Cepheid Xpert® HCV viral load. Available: https://www.cepheid. com/en/tests/Virology/Xpert-HCV-Viral-Load [Accessed 3 May 2021].

33 Augustine R, Das S, Hasan A, et al. Rapid antibody-based COVID-19 mass surveillance: relevance, challenges, and prospects in a pandemic and Post-Pandemic world. J Clin Med 2020;9. doi:10.3390/jcm9103372. [Epub ahead of print: 2110 2020].

34 Jahan F, Shamsuzzaman SM, Akter S. Diagnosis of common bacterial causes of urethritis in men by gram stain, culture and multiplex PCR. Malays J Pathol 2014;36:175-80. 
35 Nateghi Rostami M, Hossein Rashidi B, Nazari R, et al. A multiplex assay of Trichomonas vaginalis, Chlamydia trachomatis and Neisseria gonorrhoeae infections in genital specimens. J Infect Dev Ctries 2017;11:833-9.

36 Stafylis C, Bristow CC, Natoli LJ, et al. Field evaluation of a dual rapid human immunodeficiency virus and treponemal syphilis rapid test in community-based clinics in Los Angeles and new York. Diagn Microbiol Infect Dis 2019;93:325-8.

37 Whitlock GG, Gibbons DC, Longford N, et al. Rapid testing and treatment for sexually transmitted infections improve patient care and yield public health benefits. Int J STD AIDS 2018;29:474-82.

38 Widdice LE, Hsieh Y-H, Silver B, et al. Performance of the atlas genetics rapid test for Chlamydia trachomatis and women's attitudes toward point-of-care testing. Sex Transm Dis 2018;45:723-7.

39 Conway DP, Holt M, Couldwell DL, et al. Barriers to HIV testing and characteristics associated with never testing among gay and bisexual men attending sexual health clinics in Sydney. J Int AIDS Soc 2015;18:20221.

40 Fuller SS, Pacho A, Broad CE, et al. "It's not a time spent issue, it's a 'what have you spent your time doing?' issue..." A qualitative study of UK patient opinions and expectations for implementation of Point of Care Tests for sexually transmitted infections and antimicrobial resistance. PLoS One 2019;14:e0215380.

41 Harding-Esch EM, Nori AV, Hegazi A, et al. Impact of deploying multiple point-of-care tests with a 'sample first' approach on a sexual health clinical care pathway. A service evaluation. Sex Transm Infect 2017;93:424-9.

42 Mitchev N, Singh R, Naidoo J. Evaluation of the point-of-care Xpert CT/NG and OSOM Trichomonas rapid tests against the anyplexTMii STI-7 detection assay. Sex Transm Infect 2017;93:A156.

43 Mungati M, Mugurungi O, Machiha A. Performance of GeneXpert CT/NG in the diagnosis of Neisseria gonorrhoeae and Chlamydia trachomatis among men and women with genital discharge syndrome in Zimbabwe. Sex Transm Infect 2015;2:A155-6.

44 Herbst de Cortina S, Bristow CC, Joseph Davey D, et al. A systematic review of point of care testing for Chlamydia trachomatis,
Neisseria gonorrhoeae, and Trichomonas vaginalis. Infect Dis Obstet Gynecol 2016;2016:1-17.

45 Pacific Northwest Evidence-Based Practice Center. Screening for Gonorrhea and Chlamydia: Systematic Review to Update the U.S. Preventive Services Task Force Recommendations [Internet] Rockville, MD: Agency for Healthcare Research and Quality (US), 2014.

46 Lodiongo DK, K Bior B, W Dumo G, et al. Field evaluation of SD Bioline HIV/Syphilis Duo assay among pregnant women attending routine antenatal care in Juba, South Sudan. PLoS One 2018;13:e0205383.

47 McKechnie ML, Hillman R, Couldwell D, et al. Simultaneous identification of 14 genital microorganisms in urine by use of a multiplex PCR-based reverse line blot assay. J Clin Microbiol 2009;47:1871-7.

48 Rumyantseva T, Golparian D, Nilsson CS, et al. Evaluation of the new AmpliSens multiplex real-time PCR assay for simultaneous detection of Neisseria gonorrhoeae, Chlamydia trachomatis, Mycoplasma genitalium, and Trichomonas vaginalis. APMIS 2015;123:879-86.

49 Han Y, Yin Y-ping, Shi M-qin, et al. Evaluation of Abbott RealTime CT/NG assay for detection of Chlamydia trachomatis and Neisseria gonorrhoeae in cervical swabs from female sex workers in China. PLoS One 2014;9:e89658.

50 Brosh-Nissimov T, Kedem R, Ophir N, et al. Management of sexually transmissible infections in the era of multiplexed molecular diagnostics: a primary care survey. Sex Health 2018;15:298-303.

51 Muvunyi CM, Dhont N, Verhelst R, et al. Evaluation of a new multiplex polymerase chain reaction assay STDFinder for the simultaneous detection of 7 sexually transmitted disease pathogens. Diagn Microbiol Infect Dis 2011;71:29-37.

52 Choe H-S, Lee DS, Lee S-J, et al. Performance of Anyplex ${ }^{\mathrm{TM}}$ II multiplex real-time PCR for the diagnosis of seven sexually transmitted infections: comparison with currently available methods. Int J Infect Dis 2013;17:e1134-40.

53 Peeling RW. Applying new technologies for diagnosing sexually transmitted infections in resource-poor settings. Sex Transm Infect 2011;87 Suppl 2:ii28-30. 